\title{
Die Plattenosteosynthese am Humerusschaft
}

\author{
Daniel A. Rikli, Reto Babst
}

\section{Zusammenfassung}

Die Plattenosteosynthese ist eine etablierte Behandlungsmethode für Frakturen des Humerusschaftes. Die Gefahr einer Pseudarthrose ist nach Plattenosteosynthese tendenziell kleiner als nach Verwendung eines intramedullären Implantats. Hingegen ist das Risiko einer iatrogenen Nervenverletzung nach Plattenosteosynthese etwas höher als nach Marknagelung. Zugang und Lagerung des Patienten richten sich bei der Plattenosteosynthese des Humerusschaftes nach Frak- turmuster und Frakturlokalisation. In der Regel kommen Zugschrauben und Kompressionsplatten vom Typ LC-DCP zur Anwendung. Auf eine subtile Technik, sowohl im Umgang mit Weichteilen und Knochen, als auch auf genügend lange Platten für die Osteosynthese, muss geachtet werden. Das Ziel des Eingriffes muss die Wiederherstellung von Achse, Länge und Rotation sowie die stabile Fixation des Humerus sein. Diese sollte eine unmittelbar postoperativ einsetzende funktionelle Nachbehandlung der Extremität erlauben.

\section{Einleitung}

Die Plattenosteosynthese gehört neben den intramedullären Stabilisationsmethoden (Verriegelungsmarknagel, Bündelnagelung) zu den akzeptierten Verfahren der operativen Behandlung von Humerusschaftfrakturen $[4,7,8]$. Die Verfahrenswahl richtet sich dabei in erster Linie nach der Lokalisation der Fraktur und nach dem Frakturmuster, nach eventuellen Begleitverletzungen (an der gleichen Extremität, Mehrfachverletzte), nach dem allgemeinen Zustand des Patienten und oft auch nach der Erfahrung und den Vorzügen des Operateurs. Die Problematik bei der Behandlung von Oberarmschaftbrüchen ergibt sich einerseits aus den benachbarten Gelenken; hier gilt es, die Voraussetzungen für eine frühfunktionelle Nachbehandlung zur Vermeidung von Immobilisierungsschäden zu schaffen. Andererseits kennen wir Probleme bedingt durch die lokalen anatomischen und biologischen Gegebenheiten. Beidem muss bei der Wahl des Stabi-

OP-JOURNAL 2002; 18: 245-250

(c) Georg Thieme Verlag Stuttgart . New York lisierungsverfahrens und durch Anwendung einer korrekten Operationstechnik Rechnung getragen werden.

Das Ziel der operativen Behandlung ist schlussendlich die volle Funktionalität der Extremität durch Wiederherstellung von Achse, Länge und Rotation, stabile innere Fixation und frühfunktionelle Nachbehandlung. Im Vergleich zur Marknagelung ist die Pseudarthroserate nach Plattenosteosynthese tendenziell geringer ( $2 \%$ vs. $7 \%$ ), die Gefahr der iatrogenen Nervenverletzung höher (bis $17 \%$ vs. $4 \%)[2,4,6,7,8]$. Die Infektrate wird mit unter $2 \%$ angegeben $[4,8]$.

\section{Indikationen}

Die „klassische“ Plattenosteosynthese unter Verwendung von Zugschrauben und dynamischen Kompressionsplatten (DCP, LC-DCP) hat sich bei einfachen queren, schrägen und Torsionsbrüchen und bei komplexeren Brüchen mit Drehoder Biegekeil (AO-Klassifikation 12-A resp. 12-B) (Abb.1) bewährt. Die Plattenosteosynthese eignet sich aber auch zur Behandlung von Etagen-(Kompressionsplatte) und von Trümmerfrakturen (Überbrückungsosteosynthese) (AOKlassifikation 12-C) $[4,7,8]$. Bei Pseud- arthrosen des Humerusschaftes nach konservativer Therapie oder nach Marknagelung wird die Plattenosteosynthese bevorzugt $[3,9]$. Implantatwahl und $\mathrm{Zu}$ gang und damit die Lagerung richten sich nach der Lokalisation und Ausdehnung des Bruches.

\section{Implantatwahl, Zugänge, Lagerung}

\section{Proximale Schaftfrakturen}

Im proximalen Schaftdrittel finden wir Frakturen des osteoporotischen Knochens, typischerweise Biege- oder Torsionsfrakturen, meist mit einem ausgesprengten Keil. Bei knochengesunden Individuen sind Frakturen im proximalen Humerusdrittel hingegen oft die Folge von Hochrasanztraumen (Abb.2a). Sehr häufig läuft das Fraktursystem in den $\mathrm{Hu}$ meruskopf, allenfalls kombiniert mit Frakturen der Tubercula. Im letzteren Fall sind wir einerseits mit den biologischen und biomechanischen Problemen der diaphysären Fraktur, andererseits mit denjenigen der metaepiphysären Fraktur mit Beteiligung der Rotatorenmanschette konfrontiert. In dieser Situation bieten sich als mögliche Implantate neben der LC-DCP verschiedene Typen von winkelstabilen Implantaten wie die Hakenplatte (Abb.2b), die kannülierte Winkelplatte, die Kinderosteotomieplatte, die umgebogene 1/3- oder 1/2-RohrPlatte an. Mit diesen Implantaten kann auch ein kurzes Humeruskopffragment, allenfalls kombiniert mit einer Zuggurtung der Tubercula, rotationsstabil gehalten werden. Neuerdings stehen für diese Frakturen LCP-Platten mit Verriegelungsschrauben zur Verfügung. Damit besteht eine weitere Möglichkeit, das kurze proximale Humeruskopffragment mit einem winkelstabilen Implantat zu versorgen. In der Regel ist für das proximale Humerusdrittel die Dimension 3,5 mm adäquat. Der Zugang zum proximalen Humerus ist anterolateral im Sinne eines nach distal erweiterten deltoideopektoralen $\mathrm{Zu}$ ganges (Abb.3). Entsprechend wird auf 

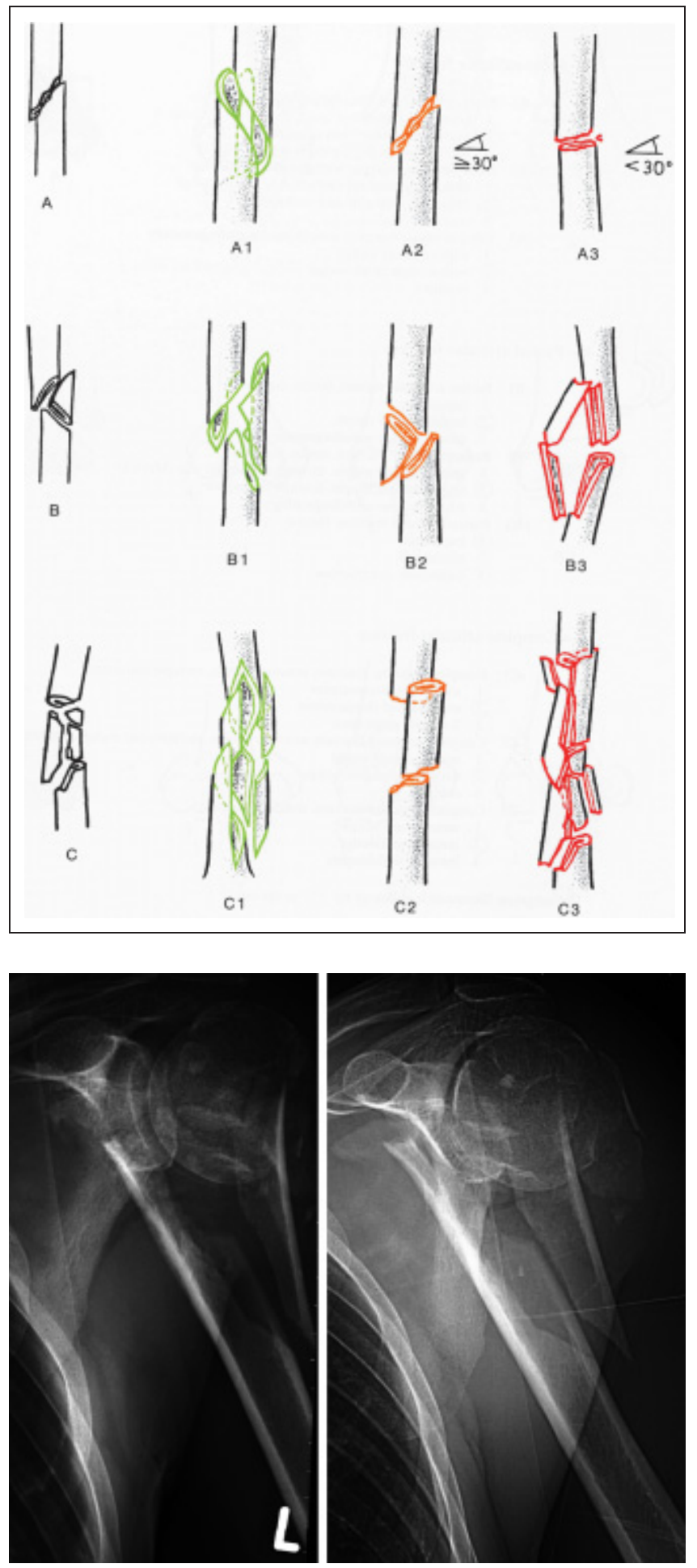

dem Schultertisch in mehr oder weniger sitzender Position gelagert (Beach chair) (Abb.4). Vor allem ältere Patienten vertragen diese Position kreislaufmäßig oft schlecht. Sie muss sich deshalb meist auf eine Inklination des Oberkörpers von ca. $30^{\circ}$ beschränken. Eine kurze Armbank resp. ein beweglicher Beistelltisch zur variablen Positionierung des Armes
Abb. 1 AO-Klassifikation der Humerusschaftfrakturen (A0 12).
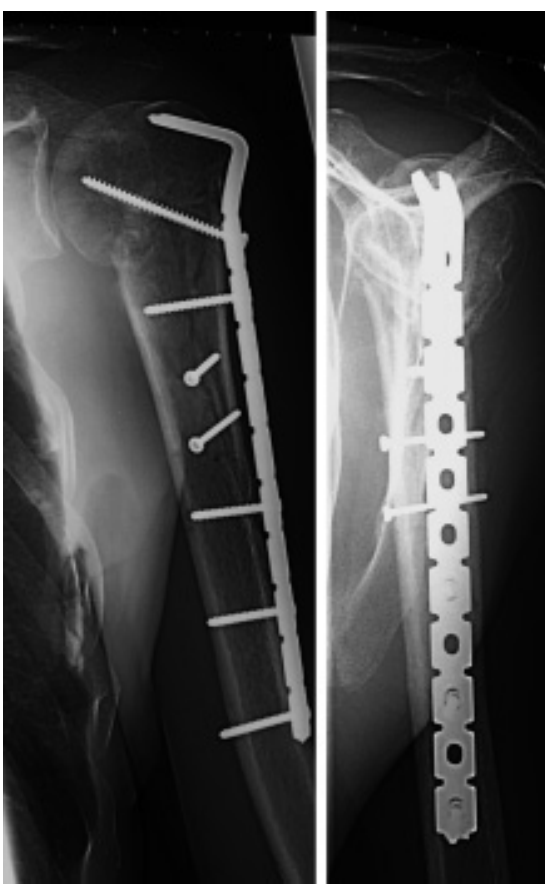

Abb. 2 b W. E. m 62 J. A0 42 B2.1, 12 w postoperativ nach Stabilisierung mit 3,5 mm-, Krallenplatte“ (3,5 mm-Rekoplatte mit Krallen).
Abb. 2a W. E. m 62 J. A0 42 B2.1, Motorradunfall li obere Extremität geschlossen.
Faszie wird eröffnet und der Raum zwischen diesen beiden Muskeln kann digital eröffnet werden. Man gelangt so auf den proximalen Humerusschaft. Die V. cephalica wird dabei vorzugsweise lateral am Deltamuskel belassen, da sie hier viele den Muskel drainierende Venen empfängt, welche somit nicht versorgt werden müssen. Je nach Frakturmuster muss auch der Subdeltoidalraum eröffnet werden, um Zugang zum Kopf und zu den Tubercula zu erhalten. Die Abduktion des Armes entspannt den Deltamuskel und erleichtert die Präparation. Die lange Bizepssehne wird identifiziert und geschont. Sie bildet eine hilfreiche Landmarke zur Einstellung der Rotation des Kopffragmentes in Relation zum Schaft. Die Präparation erfolgt nun bedarfsweise nach distal, wobei der Ansatz des Deltamuskels am Humerus partiell gekerbt wird. Ist die Präparation bis ins mittlere Schaftdrittel nötig, muss der N. musculocutaneus zwischen M. brachialis und $\mathrm{M}$. biceps brachii dargestellt und geschont werden. Bei notwendiger weitergehender Präparation nach distal ist es ratsam, den N. radalis zwischen dem M. brachialis und dem M. brachioradialis zu identifizieren. Es folgt nun die Reposition der Fraktur, vorzugsweise indirekt durch Osteosynthese des proximalen Humerus mit einem winkelstabilen Implantat, welches parallel zum Sulcus bicipitis einge- ist dabei hilfreich. Die Inzision orientiert sich proximal am Processus coracoides, distal am lateralen Rand Sulcus biciptis. Sie wird leicht geschwungen geführt ohne dabei durch die axilläre Hautfalte zu schneiden. Nach erfolgtem Hautschnitt wird die V. cephalica aufgesucht, welche das Spatium zwischen Deltamuskel und M. pectoralis major markiert. Die 

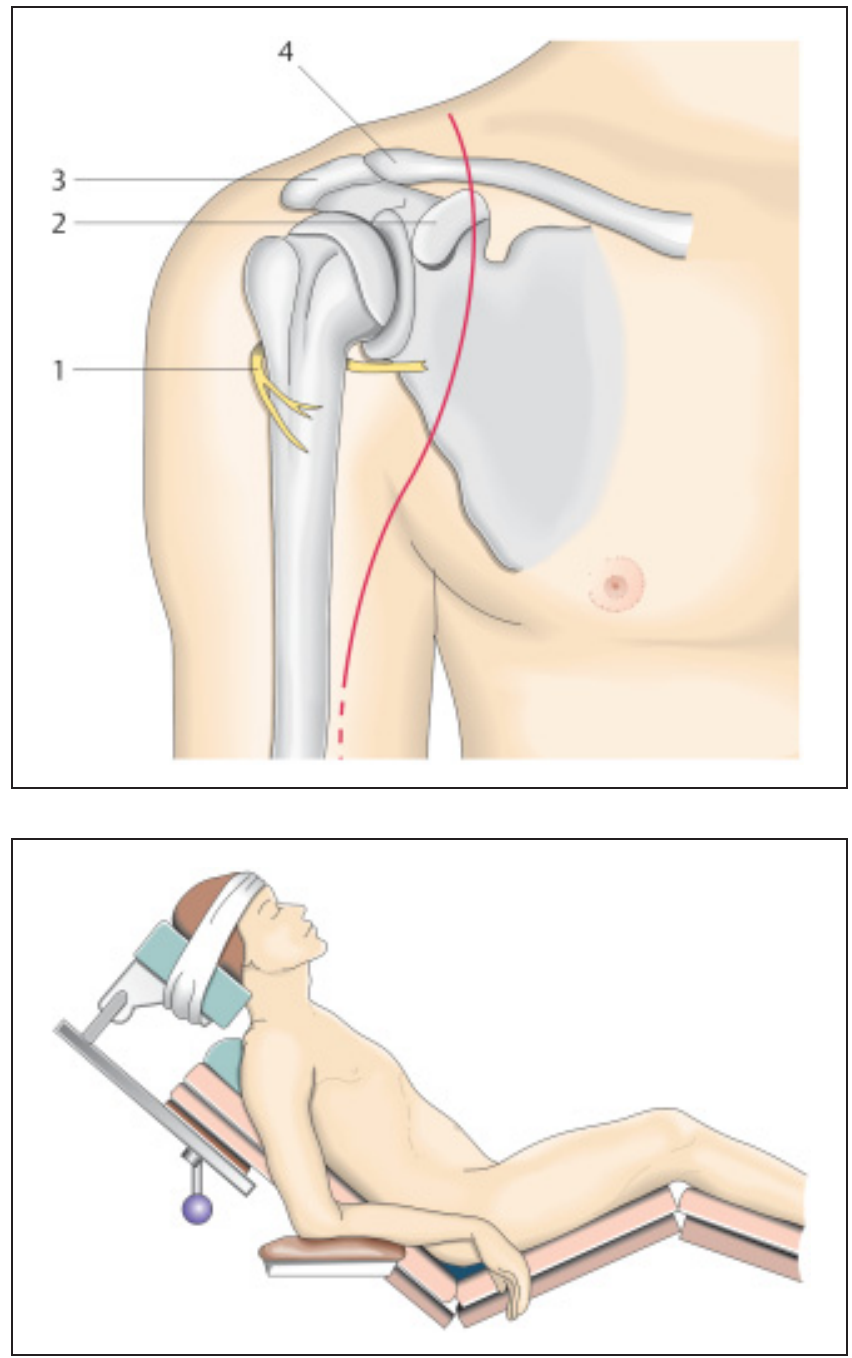

Abb. 4 Beach-chairposition für den Deltoideopektoralen Zugang.

Abb. 3 Deltiodeopektoraler Zugang.

bracht wird. Eine Trümmerzone kann dann überbrückt und gegen die distal angelegte Platte reponiert werden. Auch einfache Frakturmuster lassen sich so mit minimaler Frakturexposition retinieren (Abb.2a,b). Vor Wundverschluss erfolgt die Kontrolle von Reposition und Lage des Osteosynthesematerials mittels Bildverstärker. Für den Wundverschluss genügt in der Regel die Adaptation der Subkutis nach Legen von ein bis zwei Redon-Drainagen, gefolgt vom Hautverschluss. Meist wird für die postoperative Schmerztherapie ein Gilchrist- oder Desault-Verband oder ein Äquivalent angelegt. Unmittelbar postoperativ erfolgt die aktiv assistierte Mobilisation des Armes inkl. Schulter, Ellbogen und Hand unter Anleitung der Physiotherapie.

\section{Frakturen der Schaftmitte}

In Schaftmitte finden sich sämtliche für lange Röhrenknochen typische Frakturmuster von der Torsionsfraktur mit oder ohne Keil als Folge von simplen Stürzen durch indirekte Energieeinwirkung über Quer- und kurze Schrägfrakturen bis zu komplexen Trümmer- und Etagenfrakturen durch direktes Trauma. Immer muss eine begleitende Radialisparese ausgeschlossen werden, diese kommt am häufigsten bei Frakturen am Übergang mittleres zum distalen Drittel (s. unten) vor. Der Vorzugang richtet sich nach der Lokalisation resp. der Ausdehnung der Fraktur: Der anterolaterale Zugang (s. oben) ist nicht geeignet, wenn die Frakturstabilisierung eine Platte distal bis über die Fossa coronoidea erfordert. Der dorsale Zugang (s. unten) ist proximal limitiert durch den Ansatz des M. deltoideus. Die Lagerung richtet sich nach dem gewählten Zugang. Nach Darstellen der Fraktur und präliminärer Fixation erfolgt die Osteosynthese mit Zugschrauben und einer Kompressionsplatte (LC-DCP $4,5 \mathrm{~mm}$ schmal oder $3,5 \mathrm{~mm}$ ). Für die Verwendung einer breiten $4,5 \mathrm{~mm}$-Platte gibt es wenig biomechanische oder klini-
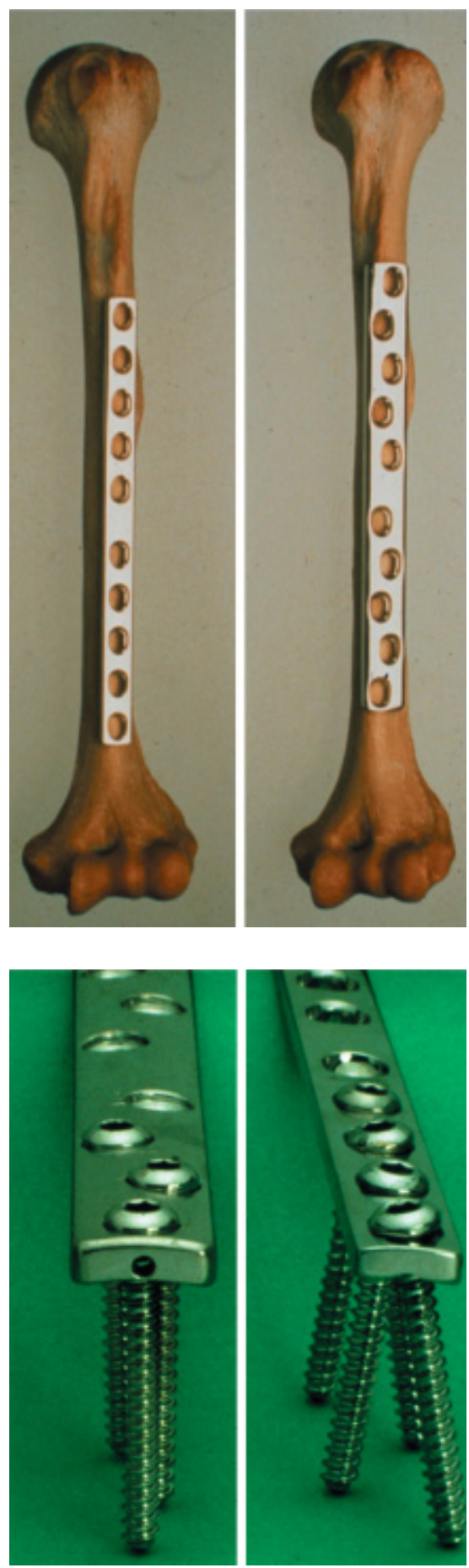

Abb. 5a u.b Die breite 4,5 mm-Platte ist für den Humerus in den meisten Fällen zu voluminös. Durch divergierendes Einbringen der Schrauben kann auch bei der schmalen 4,5 mm LC-DC-Platte eine Fissurierung der Transkortikalis - durch Schraubenspitzenpenetration in einer Linie - vermieden werden.

sche Argumente. Die Gefahr einer Schaftfissur oder -spaltung im Bereich der Transkortikalis kann durch divergierende Schraubenpositionierung (Abb.5) gebannt werden. 


\section{Distale Schaftfrakturen}

Distale Schaftfrakturen finden sich als Quer-, Schräg- oder Torsionsfrakturen mit oder ohne Keil sowohl beim jüngeren, knochengesunden als auch beim älteren Patienten mit Osteoporose. Ausläufer des Fraktursystems in die benachbarten Gelenke sind möglich und müssen entsprechend berücksichtigt werden.

Vor allem lange Torsionsfrakturen am Übergang mittleres zum distalen Schaftdrittel bergen die Gefahr einer Radialisparese. Typisch dafür sind Torsionsfrakturen mit oder ohne Drehkeil. Im distalen Drittel tritt der Nerv durch das Septum intermusculare und hat verhältnismäßig wenig Möglichkeit auszuweichen (Abb.6). Durch das Trauma selber oder die präoperative Traktion und Irritation kann hier der Nerv geschädigt werden.

Der Zugang zu diesen Frakturen ist dorsal (Abb.7), in der Regel unter Spalten der Trizepssehne. Entsprechend muss der Patient in Bauch- oder Seitenlage (Abb.8) gebracht werden, der Oberarm wird dabei vorzugsweise auf einer kurzen Armbank gelagert. Der Hautschnitt ist in der Mitte des Oberarms und orientiert sich proximal am spitzwinklig zulaufenden Ansatz des Deltamuskels, distal an der Olekranonspitze, wobei letztere bevorzugt radial leicht bogenförmig umschnitten wird. Nach Durchtrennen der Subkutis wird der Sehnenspiegel des M. triceps brachii dargestellt und in der Mitte eröffnet. Die Muskelfasern werden stumpf

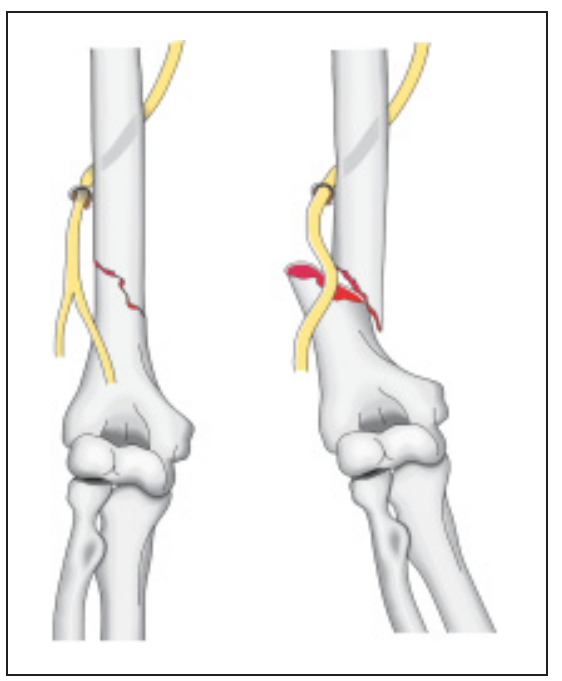

Abb. 6 Torsionsfraktur im distalen Humerusschaftdrittel (Holstein-Lewis-Typ). Gefahr einer Verletzung des N. radialis, welcher auf dieser Höhe durch die Membrana interossea hindurchtritt und wenig Ausweichmöglichkeiten hat.

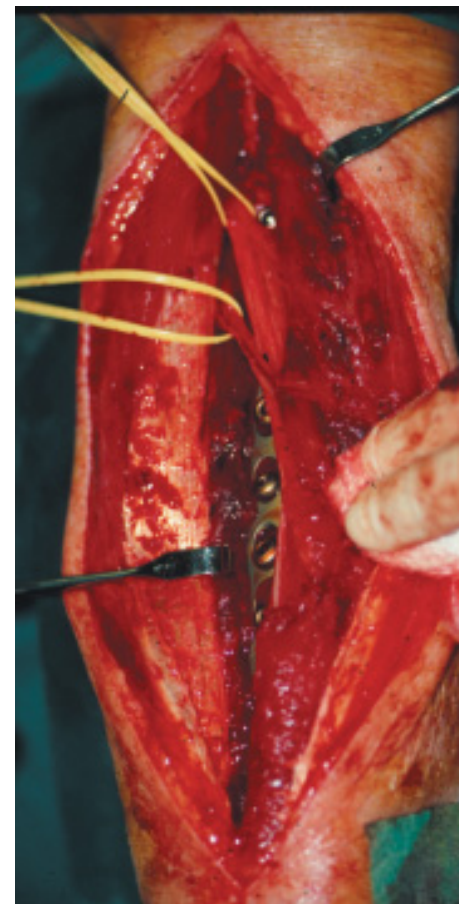

Abb. 7 Dorsaler Zugang zum Humerus. Der Patient muss dafür entweder auf dem Bauch oder in Seitenlage gelagert sein. Der N. radialis ist angeschlungen und die Plattenspitze liegt unter dem $\mathrm{N}$. radialis.

auseinander gedrängt bis auf den Humerusschaft. Proximal stößt man auf das Caput mediale, an dessen Oberrand verläuft der $\mathrm{N}$. radialis als flache, relativ breite Struktur (Abb.8). Er muss gezielt aufgesucht, dargestellt und schonend unterfahren werden. Nun wird die Fraktur reponiert, präliminär fixiert und die Osteosynthese mittels Zugschrauben und einer Platte mit minimal 3 Löchern jenseits der Fraktur komplettiert (Abb.9b). Je nach Frakturmuster muss die Platte auf den radialen Pfeiler des distalen Humerus gezogen werden, um die Fossa olecrani nicht zu obstruieren. Die Platte kommt dann auf dem Humerusschaft proximal etwas schräg zu liegen. Nach Kontrolle von Reposition und Implantatlage mit dem Bildverstärker erfolgt der schichtweise Wundverschluss über Redondrainagen.
Der Trizepssehnenspiegel wird mit einem resorbierbaren Faden verschlossen. Zur Schmerztherapie während der ersten Tage postoperativ wird ein Gilchrist- oder Desault-Verband oder ein Äquivalent angelegt. Unmittelbar postoperativ erfolgt die Mobilisation des Armes inkl. Schulter, Ellbogen und Hand unter Anleitung der Physiotherapie.

\section{Komplikationen}

\section{Pseudarthrosen}

Knöcherne Heilungsstörungen im Sinne von verzögerter Heilung bis hin zur Pseudarthrose kommen vor allem bei Quer- und kurzen Schrägfrakturen vor. Die Inzidenz ist aber bei technisch korrekt ausgeführter Osteosynthese und schonendem Umgang mit den Weichteilen deutlich geringer als bei einer Marknagelosteosynthese (2\% vs. $7 \%$ ) $[4,5,6,7,8]$.

Bleibt am Humerusschaft die Frakturüberbrückung während 4-8 Monaten aus, so spricht man von einer verzögerten Heilung (delaved union). Wird dieser Zeitpunkt überschritten, handelt es sich um eine Pseudarthrose.

Diese findet sich im Schaftbereich, vor allem nach kurzen Schräg- und Querfrakturen, bei fehlendem Fragmentkontakt durch Weichteilinterposition oder nach ungenügender Osteosynthese. Das Behandlungskonzept richtet sich nach Lokalisation und Art der Pseudarthrose. Ist die Ursache „biologisch“ aufgrund unzureichender Vaskularisation resp. Reagibilität des Knochengewebes (atrophe P.), braucht es neben der stabilen Versorgung der Fraktur zusätzlich einen biologischen Stimulus, in der Regel in Form einer Spongiosaplastik. Stehen biomechanische Ursachen im Vordergrund (hypertrophe, normotrophe P. [Abb.10a]), braucht es in der Regel lediglich eine stabile Osteosynthese mit Kompression im ehemaligen Frakturbereich (Abb.10b), damit die Frakturheilung zustande

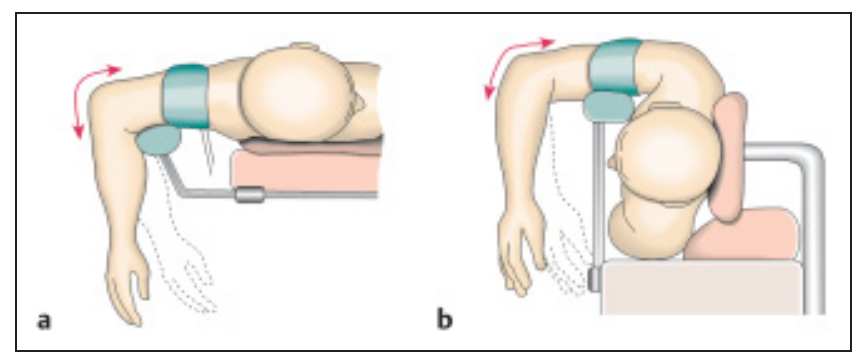

Abb. 8 Positionierung des Patienten in Bauch- oder Seitenlage 

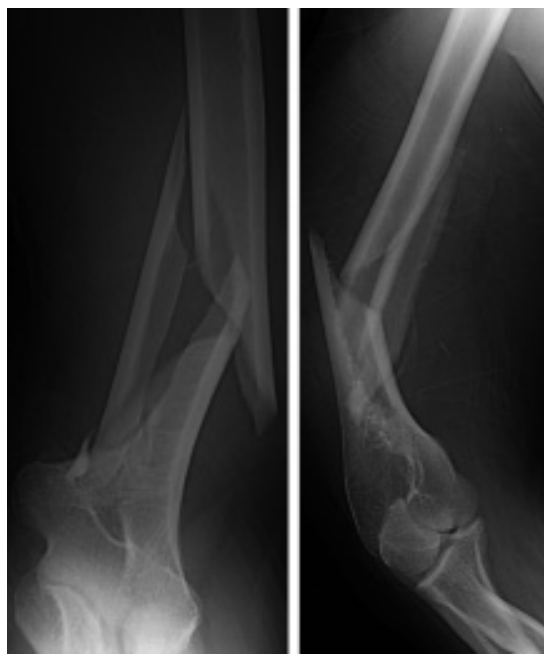

Abb. 9a E. G. m 46 ]; Ao B 2.3. Geschlossene Fraktur im distalen Drittel mit Drehkeil ohne neurologische Ausfälle.
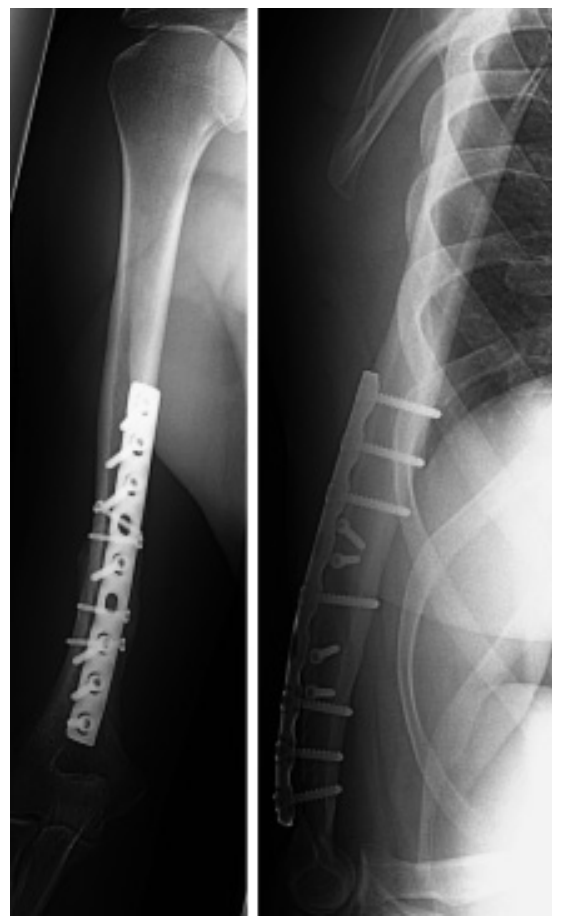

Abb. 9 b E. G. m 46 J; A0 B 2,3 B. W. St 46 J m. A0 12 A2.2; 12 Wochen nach Osteosynthese mit schmaler 4,5 mm-LC-DB-Platte plattenabhängiger und -unabhängigen $(3,5 \mathrm{~mm})$ Zugschrauben.

kommt $[3,9]$. Eine Spongiosaplastik ist unter diesen Bedingungen selten nötig.

\section{Radialisparese}

Radialisparesen - sie äußern sich klinisch mit einer „Fallhand“ (die Hand kann nicht extendiert werden) und Sensibilitätsstörungen im Bereich des Handrückens können unmittelbar posttraumatisch
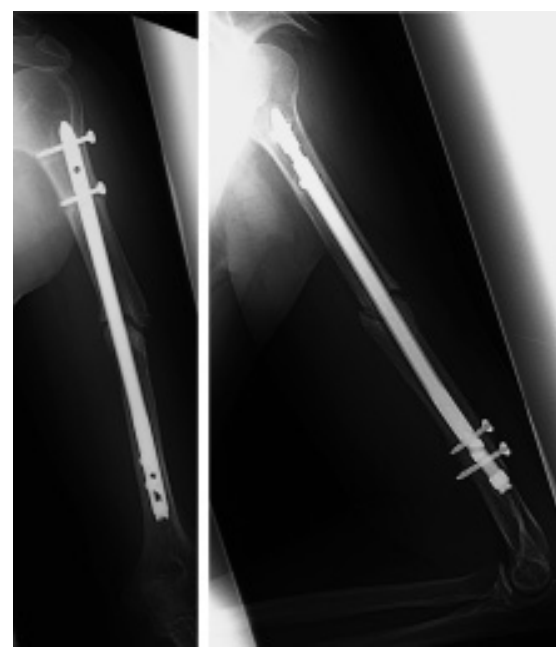

Abb.10a $\quad$ B. S. m 41 J; Hypotrophe Pseudarthrose, 8 Monate nach verriegeltem Humerusnagel bei kurzer geschlossener Schrägfraktur A0 12 A 3.
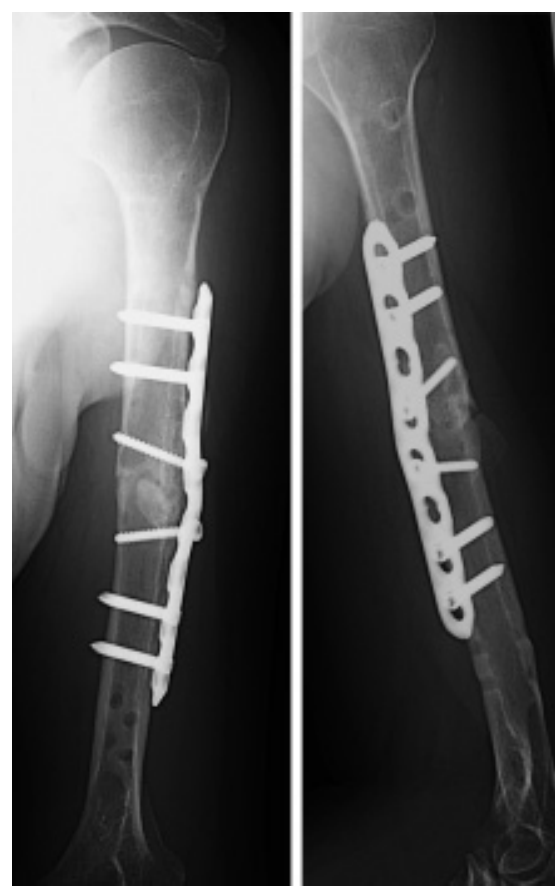

Abb.10b B. S. m 41 J; 12 w nach Kompressionsplattenosteosynthese mit LC-Platte.

auftreten, aber auch verzögert z.B. im Rahmen einer konservativen Behandlung und nach operativer Stabilisierung (nach dorsalen, aber auch nach anterolateralem Zugang).

Die Inzidenz posttraumatischer Radialisparesen kann je nach Schwere der Verletzung bis zu $35 \%$ betragen [5]. Am häufigsten ist der Nerv posttraumatisch bei Frakturen am Übergang mittleres zum distalen Drittel paralysiert („Holstein-Lewis“-Typ der Fraktur (vgl. Abb.6 u.9a).
Meist handelt es sich dabei um Traktionsschäden oder direkte Irritationen durch die Knochenfragmente, nur selten ist der Nerv eingeklemmt oder gar durchtrennt. Tritt die Parese sofort oder verzögert nach dem Unfall auf, sollte der Nerv revidiert werden $[1,6,10]$. Die Fraktur sollte zur Vermeidung weiterer Nervenschädigung stabilisiert werden. Vorzugsweise wird hier die Plattenosteosynthese gewählt, da gleichzeitig der Nerv revidiert werden kann.

Postoperative Radialisparesen nach Plattenosteosynthese treten nach Literaturangaben zwischen $2 \%$ und $17 \%[4,5,8]$ auf. Tritt die Parese postoperativ auf, erübrigt sich die Revision, falls der Nerv intraoperativ dargestellt wurde. Die Erholungstendenz ist vor allem beim jüngeren Individuum gut. Posttraumatische Paresen erholen sich in etwa $80 \%$ partiell oder vollständig. Paresen nach Plattenosteosynthese sind in fast $100 \%$ regredient $[4,5]$.

Beim anterolateralen Zugang kann der N. musculocutaneus zusätzlich Schaden nehmen. Er sollte daher immer dargestellt und geschont werden. Die Parese äußert sich durch einen motorischen Ausfall von M. biceps brachii und M. brachialis (Ellbogenflexion, Supination) und Sensibilitätsstörungen im Bereich des Vorderarms (N. cutaneus antebrachii lateralis).

\section{Infektionen}

Sie sind glücklicherweise bei geschlossenen Frakturen nach Plattenosteosynthesen selten. Die Inzidenz ist unter $2 \%$ $[4,8]$. Offene Frakturen am Humerusschaft sind selten. Dank dem gut durchbluteten Weichteilmantel sind Infekte nach offenen Frakturen ebenfalls rar.

\section{Implantatversagen}

Plattenbruch oder Implantatausrisse sind selten. Sie resultieren meist aus operationstechnischen Fehlern (Implantat zu kurz, zu kleine Dimensionierung) und sie finden sich vor allem bei Patienten mit schlechter Knochenqualität (Osteoporose) [4].

\section{Complex Regional Pain Syndrome (CRPS) oder Algodystrophie}

Vor allem bei gleichzeitig vorliegenden weiteren Frakturen der gleichen Extremität („Kettenverletzung“) kommt es nicht selten zu mehr oder weniger ausgeprägten lokalen trophischen Störungen ver- 
bunden mit ausgeprägten Schmerzzuständen (CRPS oder Algodystrophie, hier auch „Schulter-Arm-Syndrom“ genannt). Dieses Syndrom kann vollständig ausheilen, kann aber auch mit bleibenden Schäden, insbesondere Bewegungseinschränkungen im Bereich von Schulter, Ellbogen und Hand inkl. Finger einhergehen. Auch ohne ein eigentliches CRPS können Bewegungslimits zurückbleiben.

\section{Technische Aspekte}

Zugänge müssen in guter Kenntnis der Anatomie subtil und atraumatisch entwickelt werden. Die Durchblutung des Knochens soll geschont, eine unnötig großzügige Darstellung des Frakturgebietes und einzelner Fragmente mit dadurch zwangsläufig erfolgender Devaskularisation soll vermieden werden. Grobe Repositionszangen sind bei Verwendung indirekter Repositionstechniken kaum mehr nötig zur temporären Retention der Fraktur. Bei Quer-, Schräg- und Torsionsfrakturen ist die Kompressionsplatten-Osteosynthese indiziert. Die Grundprinzipien dieser Plattenosteosynthese bestehen im Vorbiegen der Platte sowie in der Verwendung von plattenunabhängigen oder Platten-Zugschrauben und exzentrischen Plattenschrauben. Damit wird „absolute Stabilität" erreicht, die Voraussetzung für eine direkte Knochenheilung (Abb. 9b und $\mathbf{1 0 b}$ ). Die LC-DC-Platte muss exakt angepasst sein, ansonsten kann es auch bei schon korrekt liegenden Zugschrauben zu einer Verwerfung der Fraktur kommen.

Als Faustregel sollten pro Hauptfragment 6 Corticales (3 bikortikale Schrauben) gefasst sein. Bei Trümmerfrakturen ist „absolute Stabilität“ durch eine Kompressionsosteosynthese nicht möglich, die Frakturzone wird vielmehr mit einer Platte überbrückt („relative Stabilität“). Um die Kräfte, welche durch die Schwingung im Frakturbereich entsteht, genügend zu verteilen, sollten längere Platten mit 3 bikortikalen Schrauben pro Hauptfragment verwendet weden. Die bevorzugte Dimension ist die schmale 4,5 mm-LC-DCP-Platte, bei zierlichen Individuen die $3,5 \mathrm{~mm}$-LC-DCP-Platte.

Die neue Plattengeneration der Locked Compression Plates (LCP) bietet den Vorteil, den winkelstabilen Platten-Schraubenverbundes mit der Kompressionsplattentechnik zu kombinieren (Abb.10b). Bei anatomischer Reposition und interfragmentärer Kopression des Schaftes entsteht durch die Auflage der als Fixateur wirkenden LCP-Platte keine Verwerfung durch die nicht absolut anatomisch angepasste Platte.

Zudem wird durch den nicht mehr notwendigen Anpressdruck der LCP-Platte die Vaskularisation des Knochens unter der Platte nicht mehr kompromitiert. Der Stellenwert dieser neuen Implantatgeneration wird sich innerhalb der nächsten Jahre zeigen.

\section{Nachbehandlung}

Das Ziel jeder Plattenosteosynthese muss das Erreichen einer übungsstabilen inneren Fixation der Fraktur sein. Entsprechend erfolgen Mobilisation von Schulter, Ellbogen und Hand unmittelbar postoperativ aktiv-assistiv unter physiotherapeutischer Anleitung. Bis zum Kontrollröntgenbild nach 6 Wochen wird die betroffene Extremität unbelastet mobilisiert. Der Belastungsaufbau richtet sich nach dem radiologisch dokumentierbaren Heilungsverlauf. Er beginnt bei ungestörter Knochenheilung in der Regel nach 8-10 Wochen.

\section{Implantatentfernung}

Nur selten ist eine Metallentfernung an der oberen Extremität notwendig. Indiziert ist sie beim wachsenden Skelett oder bei lokaler Irritation zwischen Material und Weichteilen, bei Infekt und Implantatversagen.

Wegen der Gefahr einer Verletzung des N. radialis resp. N. musculocutaneus anlässlich der Metallentfernung muss die Indikation zur Osteosynthesematerialentfernung, insbesondere bei dorsaler Plattenlage (N. radialis überkreuzt die Platte) sehr kritisch gestellt werden.

\section{Schlussfolgerung}

Die Plattenosteosynthese am Humerusschaft hat sich bei gegebener Operationsindikation als sicheres Verfahren erwiesen. Komplikationen sind bei Anwendung atraumatischer Repositions- und Retentionstechniken, bei korrekter Implantatwahl (Plattendimension und -länge) weitgehend vermeidbar. Genaue Kenntnis der Anatomie und schonende Operationstechnik verhindern postoperative Paresen des N. radialis. Die Prognose bezüglich der Erholung des Nerven ist, insbesondere beim jungen Patienten, gut.
Für die Behandlung von Humerusschaftpseudarthrosen bleibt die Plattenosteosynthese der „Gold Standard“.

\section{Literatur}

${ }^{1}$ Alnot J, Osman N, Masmejean E, Wodecki P. Lesions of the radial nerve in fractures of the humeral diaphysis. Apropos of 62 cases. Rev Chir Orthop Reparatrice Appar Mot 2000; 86(2): $143-50$

${ }^{2}$ Blum J, Rommens PM, Janzing H, Langendorff HS. Retrograde nailing of humerus shaft fractures with the unreamed humerus nail. An international multicenter study. Unfallchirurg 1998 May; 101(5): 342-52

${ }^{3}$ Devnani AS. Simple approach to the management of aseptic non-union of the shaft of long bones. Singapore Med J 2001 Jan; 42(1): $020-5$

${ }^{4}$ Heim D, Herkert F, Hess P, Regazzoni P. Can humerus shaft fractures be treated with osteosynthesis? Helv Chir Acta 1992 Mar; 58(5): $673-8$

${ }^{5}$ Lim KE, Yap CK, Ong SC, Aminuddin. Plate osteosynthesis of the humerus shaft fracture an its association with radial nerve injury - a retrospective study in Melaka General Hospital. Med J Malaysia 2001 Jun; 56 Suppl C: $8-12$

${ }^{6}$ Mauch J, Renner N, Rikli D. Intramedullary nailing of humeral shaft fractures - initial experiences with an unreamed humerus nail. Swiss Surg. 2000; 6(6): 299-303

${ }^{7}$ McCormack RG, Brien D, Buckley RE, McKee MD, Powell J, Schemitsch EH. Fixation of fractures of the shaft of the humerus by dynamic compression plate or intramedullary nail. A prospective, randomised trial. J Bone Joint Surg BR 2000 Apr; 82(3): 336-9

8 Paris H, Tropiano P, Clouet D'orval B, Chaudet H, Poitout DG. Fractures of the shaft of the humerus: systematic plate fixation. Anatomic and functional results in 156 cases and a review of the literature. Rev Chir Orthop Reparatrice Appar Mot 2000 Jun; 86(4): 346-59

${ }^{9}$ Rubel IF, Kloen P, Campbell D, Schwartz M, Liew A, Myers E, Helfet DL. Open reduction and internal fixation of humeral nonunions: a biomechanical and clinical study. J Bone Joint Surg Am 2002 Aug; 84-A(8): 1315-22 ${ }^{10}$ Takami H, Takahashi S, Ando M. Severance of the radial nerve complicating transverse fracture of the mid-shaft of the humerus. Arch Orthop Trauma Surg 1999; 119(1-2): 109-11

Dr. med. Daniel A. Rikli Leitender Arzt Unfallchirurgie

PD Dr. med. Reto Babst

Co-Chefarzt und Leiter der Unfallchirurgie

Chirurgische Klinik A

Kantonsspital Luzern

Spitalstrasse

CH-6002 Luzern 